\title{
Optimal maintenance planning for sustainable energy efficiency lighting retrofit projects by a control system approach
}

\author{
Xianming Ye*, Xiaohua Xia, Lijun Zhang, Bing Zhu \\ Department of Electrical, Electronic and Computer Engineering, University of Pretoria, Pretoria 0002, South Africa
}

\begin{abstract}
The energy savings achieved by implementing energy efficiency (EE) lighting retrofit projects are sometimes not sustainable and vanish rapidly given that lamp population decays as time goes by if without proper maintenance activities. Scope of maintenance activities refers to replacements of failed lamps due to nonrepairable lamp burnouts. Full replacements of all the failed lamps during each maintenance interval contribute to a tight project budget due to the expense for the lamp failure inspections, as well as the procurement and installation of new lamps. Since neither "no maintenance" nor "full maintenance" is preferable to the EE lighting project developers (PDs), we propose to design an optimal maintenance plan that optimises the number of replacements of the failed lamps, such that the EE lighting project achieves sustainable performance in terms of energy savings whereas the PDs obtain their maximum benefits in the sense of cost-benefit ratio. This optimal maintenance planning (OMP) problem is aptly formulated as an optimal control problem under control system framework, and solved by a model predictive control (MPC) approach. An optimal maintenance plan for an EE lighting retrofit project is designed as a case study to illustrate the effectiveness of the proposed control system approach.
\end{abstract}

Keywords: Energy Efficiency, Optimal Control, Lighting, Maintenance Planning, MPC

\section{Introduction}

Lighting is the first service offered by electric utilities and continues to be one of the largest electrical end-uses. For 2005 it is estimated that grid-based electric lighting consumed 2651 Tera Watt hour (TWh) of electricity, which is $19 \%$ of global electricity consumption [12]. Past research has shown that a great potential of energy savings can be generated with the energy efficiency (EE) solution of lighting retrofit $[18,12,16]$. The lighting retrofit approach is to replace inefficient lamps with efficient ones.

Due to the great savings potential of lighting energy usage, a large number of lighting retrofit projects have been implemented under various incentive EE programmes such as clean development mechanism (CDM) [17], white tradable certificate (WTC) scheme [20,1], demand side management (DSM) programmes [9], and performance contracting [19]. However, maintenance has not been suitably addressed in most of the existing lighting projects $[21,29,30]$. And no maintenance activities are required for the implemented lighting projects in the CDM lighting guidelines [25, 26]. For these "no maintenance" lighting projects, the service level of the installed EE lighting devices will be deteriorated due to the following lighting failure factors, i.e., flickering, lumen depreciation caused by age

\footnotetext{
${ }^{2}$ A preliminary version of this paper has been presented in the 19th World Congress of the International Federation of Automatic Control, Cape Town, South Africa, 24-29 August 2014.

${ }^{*}$ Corresponding author. Tel.: +27 (0)12 420 4353; fax: +27 (0)12 362 5000; Email address: xianming.ye@up.ac.za (Xianming Ye).
}

or dirt, lamp burnouts or ballast failures as time goes by. These lighting failure factors will consequently cause deceases of both the lighting project population and performance. To deal with the lamp population decay of these EE lighting projects, the guidelines [25, 26] apply a penalise factor, which is called lamp failure rate (LFR) to the energy savings calculation and further restrict that no credits will be issued to the implemented projects when $50 \%$ of the initial population is failed during the project crediting period. Under these rules, although lighting projects are allowed a crediting period of 10 years, most of these projects only obtain rebates for the first couple of years due to the lamp failures [29, 30]. The EE lighting projects are only considered sustainable when the survived lighting population is equal to or greater than $50 \%$ of their initial population by proper maintenance. To this end, some latest designed lighting project guidelines $[27,28]$ request to perform continuous replacements of all the failed lamps. Practically, the following barriers hold the project developers (PDs) back from performing such full maintenance policy. Firstly, the maintenance activities can only be carried out when the project device failures are observed during the project inspections. However, continuously monitoring and sampling the lighting devices' working conditions are very costly and time-consuming when large decentralised lamp population is involved. Secondly, the maintenance activities also require additional investments for the procurement and installation of the new lighting devices. The extra investments sometimes contribute to a tighter project budget.

Since neither the "no maintenance" nor the "full maintenance" policy is preferable to the PDs, it is thus interesting to 
find an "optimal maintenance" policy that contributes to a sustainable energy/cost savings whilst the PDs obtain their maximum financial benefits in the sense of the cost-benefit ratio by optimising the maintenance actions and schedules. The optimal maintenance planning (OMP) problem can be aptly formulated under the control system framework and solved by control system approaches. In the literature, the control system approach has been adopted to deal with similar OMP problems for various commercial and industrial systems. For instance, scheduling of periodic maintenance for transportation equipments is accomplished by a fuzzy control system approach in [10]. Ref. [14] outlines six types of decisions to design the optimal maintenance strategy as part of the entire control system optimisation. The principle component analysis (PCA) approach has been used in [15] that helps the prediction of the type and time of future device failures, which also contributes to the optimal scheduling of maintenance work. In [13], it designs optimal maintenance policies based on impulse control models in which the optimal actions and schedules are optimised for a compound Poisson shock model. In addition, the optimal control and stochastic control approaches are applied respectively in [3] and [4] to assist the planning of production and maintenance in a flexible manufacturing system.

The control system framework is also applicable in this study since the population dynamics of the EE lighting projects are characterised and modelled as state space equations. The lamp population decay dynamics of the project are taken as the plant of the control system. Practically, the failure dynamics of the EE lamps vary from different individuals due to different technical specifications, working conditions and operating schedules. In order to simplify the modelling complexities but without loss of generality, it is assumed that the lighting project population be classified into several homogeneous groups, where devices in the same group are of the same technical specification (i.e., model, make, rated power, life span, etc.), the same operating schedule and working condition. Consequently, lighting devices from the same group are deemed to have the same energy saving and economic performance, and the same population decay dynamics. In this case, the state variables can be chosen as the survived lighting population in each homogeneous group instead of the working/fail status of individual lighting devices. In order to achieve sustainable energy savings and maximum project profits, it is recommended to optimally control/replace a number of failed lighting devices during each maintenance interval. The number of failed lamps to be replaced is taken as the control variable of the control system. As different lighting technologies have different population decay dynamics and different rebate tariffs, the control inputs can be optimally decided based on the PDs' budget availability.

Bringing the OMP problem of the EE lighting projects into the control system framework exhibits the following advantages. Firstly, the OMP problem can be formulated as an optimal control problem. Optimal solutions to this problem determine the optimal maintenance policy, with which sustainable energy savings are maintained whilst the maximum project profit is achieved in terms of the cost-benefit ratio. Secondly, classic control theories and methodologies can be applied to further improve the designed maintenance strategy. In this study, model predictive control (MPC) approach is introduced to solve the OMP problem as it converges to the optimal solution fast and is advantageous in dealing with the control system uncertainties and disturbances with the establishment of a closed-loop control system [33, 23]. An optimal maintenance plan for an EE lighting retrofit project is designed as a case study to illustrate the effectiveness of the proposed control system approach. In addition, multiple simulations are carried out to test the applicability of the proposed model to other similar lighting projects with different rebate tariffs, different lighting device life spans, and different unit retrofit prices. The case study and the simulation results suggest that the proposed optimal control model is widely applicable to other similar projects.

The rest of this paper is organised as follows: in Section 2, the OMP problem is mathematically formulated under control system framework as an optimal control problem. Then in Section 3, the MPC approach is introduced to solve the OMP problem. Afterwards, an optimal maintenance strategy is designed in Section 4 for an EE lighting project as a case study to illustrate the effectiveness of the proposed control system approach. Thereafter, multiple simulations results based on the case study are also presented in Section 5 to further explore the potential strength and weakness of the proposed approach. Remarks on the current study and future work are raised at the end.

\section{Problem formulation}

In this section, the OMP problem is mathematically formulated, followed by discussions on the maintenance policy and lamp population decay dynamics.

\subsection{Maintenance policy for lighting projects}

In order to design optimal maintenance plans for EE lighting projects, the most suitable maintenance policy that covers both the maintenance actions and schedules needs to be properly selected. As defined in MIL-STD-721C [8], maintenance actions refer to retain an item in or restore it to a specified condition. Maintenance actions can be classified by two major categories: preventive maintenance (PM) and corrective maintenance (CM), where PM means all actions performed in an attempt to retain an item in specified condition by providing systematic inspection, detection, and prevention of incipient failures and CM refers to all actions performed as a result of failure, to restore an item to a specified condition [31, 22, 8]. PM is commonly carried out at fixed time intervals to improve the availability or to extend the life of the system while CM is performed at unpredictable intervals as the occurrence of failure cannot be known a priori [13]. In the literature, massive maintenance policies have been proposed with cost-effectively maintenance actions and schedules. These policies are well summarised in $[31,22]$ in terms of single-unit system maintenance policies and multi-unit system maintenance polices. Particularly, the age-dependent PM policy, periodic PM policy, failure limit policy, sequential PM policy and repair limit policy 
are specilised for the single-unit systems while the group maintenance policy and opportunistic maintenance policy are most applicable to the multi-unit systems.

As commented in [31], the aforementioned maintenance policies are sometimes applied in combination in order to obtain "global" optimal cost savings. However, the maintenance policy should not be designed too complicated to cause inconvenience in implementation in practice. For the EE lighting projects with large population, the periodic PM policy is most practical since it neither leads to unequal maintenance intervals, nor requires records on the unit usage and age. From the PDs' point of view, the principle maintenance objective of running the EE lighting project is no longer for longevity of individual lighting device but for sustainable project performance and rebates. Thus on the device level, the CM that refers to direct replacements of the failed lamps is considered on occurrence of lamp failures. On the project level, it is more feasible to perform PM by replacing part of failed lamps at certain maintenance intervals, in order to maintain the lighting project population between $50 \%$ and $100 \%$. The $\mathrm{CM}$ to the entire project refers to the "full maintenance" policy that is sometimes not applicable due to the budget and/or time constraints.

In summary, the most applicable maintenance philosophy for the EE lighting projects is the periodic group preventive maintenance. The periodic PM will be performed at fixed intervals in terms of different countable time intervals such as hourly, daily, weekly, monthly or yearly, depending on the importance of the studied lighting systems. For instance, for the general lighting services in residential sectors, PDs may be allowed to perform the maintenance actions on annual basis. One may argue that negative impacts of not replacing failed lamps may be incurred since users no longer have adequate lighting to perform necessary tasks. In practice, this valid concern is suitably addressed by allowing the users to replace the failed lamps themselves but excluding the rebate for such replacements from the PDs' benefits. However, for lighting projects with critical lighting systems, such as traffic lights, surgery lighting systems, the maintenance actions must be performed more frequently to ensure the required lighting service level. Based on the selected maintenance philosophy for the EE lighting projects, the rest of the paper will focus on the optimisation of the number of failed lamps to be replaced at fixed maintenance intervals.

\subsection{OMP problem formulation under control system frame- work}

The OMP problem is formulated under control system framework in this subsection. Given a lighting retrofit project with $I$ kinds of EE devices involved, then each kind of EE devices can be classified into the same lighting group when the $i$ th lighting group exhibits the same lighting technology, same operating schedule and working condition. Let $t_{0}$ and $t_{f}$ denote the beginning and end of the project crediting period, respectively. Once the project crediting period $\left[t_{0}, t_{f}\right]$ and the maintenance schedules are determined, $t_{k}=t_{0}+k T, k=0,1, \ldots, K-1$ is used to denote the time intervals for the maintenance, where $T$ is a constant to represent the fixed maintenance interval. When time sequence $\left\{t_{k}\right\}$ and $T$ are both determined, $t_{k}$ can be simply denoted by $k$ and the time period $\left[t_{k}, t_{k+1}\right)$ is simplified as $[k, k+1) . \quad x_{i}(0)$ denotes the quantity of the initial installation of the EE lighting devices in the ith group. Generally, the lighting project OMP problem is to find the optimal control sequences $\mathbf{u}(k)=\left[u_{1}(k), u_{2}(k), \ldots, u_{I}(k)\right]^{T}$ within the time period $[0, K)$. Here $u_{i}(k)$ is the control system input, which is the number of replacements of the failed lamps during the interval $[k$, $k+1)$ in the $i$ th group. Then the OMP problem under the control system framework is formulated in the following general form:

$$
\left\{\begin{array}{l}
\mathbf{x}(k+1)=\mathbf{f}(\mathbf{x}(k))+\mathbf{u}(k)+\mathbf{w}(k), \\
\mathbf{y}(k)=\mathbf{x}(k)+\mathbf{v}(k),
\end{array}\right.
$$

where $\mathbf{x}(k)=\left[x_{1}(k), x_{2}(k), \ldots, x_{I}(k)\right]^{T}$, denotes the state variable that corresponds to the number of survival EE devices for the time interval $[k, k+1)$ in the $i$ th group. The system output $\mathbf{y}(k)$ is the measurements of $\mathbf{x}(k)$, more precisely, $y_{i}(k)$ is the sampling result of $x_{i}(k)$ at time $k$ in the ith group. $\mathbf{f}(\mathbf{x}(k))$ denotes the function to characterise the project population decay dynamics. In addition, $\mathbf{w}(k)=\left[w_{1}(k), w_{2}(k), \ldots, w_{I}(k)\right]^{T}$ and $\mathbf{v}(k)=\left[v_{1}(k)\right.$, $\left.v_{2}(k), \ldots, v_{I}(k)\right]^{T}$ denote the modelling uncertainties and measurement disturbances, respectively.

\subsection{Lighting population decay dynamics modelling}

In order to solve the OMP problem, the lighting population decay dynamics model $\mathbf{f}(\mathbf{x}(k))$ needs to be characterised. The CDM guideline [26] has proposed a linear lamp population decay model, which is widely used for CDM projects. However, this model is not good enough to characterise the lamp population decay dynamics as it assumes a constant hazard rate of the EE lighting devices $[6,5]$. The studies $[6,5]$ offer an informative review on the existing lamp population decay dynamics models as can be found in $[21,2]$. In addition, $[6,5]$ also proposed a general form of the population decay dynamics model by re-calibrating existing models established from biological population dynamics study or from reliability engineering experiments. The general form of the model is provided in Eq. (2).

$$
s(t)=\frac{1}{c+a e^{b t}}
$$

where $s(t)$ is the percentage of survived devices at time $t$ for a lighting project, $t$ is counted from the completion of the EE lighting project implementation. $a=e^{-L}$ and $L$ is the rated average life span of a certain model of the EE devices. The rated average life span is declared by the manufacturer or responsible vendor as being the expected time at which $50 \%$ of any large number of EE devices reach the end of their individual lives [26]. $b$ is the slope of decay and $c$ is the initial percentage lamp survival at $t=0$. Thus, with a given $L, b$ and $c$ can be obtained by solving the following equations:

$$
\left\{\begin{array}{l}
s(0)=1 \\
s(L)=0.5 .
\end{array}\right.
$$

The discrete and dynamic form of model (2) is also given in $[6,5]$ as follows

$$
s(k+1)=\tilde{b} \tilde{c} s(k)^{2}-\tilde{b} s(k)+s(k),
$$


where $s(k)$ is the survived percentage of the lighting project population at the $k$ th sampling interval. Note that for different EE lighting devices, the parameters $\tilde{b}$ and $\tilde{c}$ are different and they can be obtained by the system identification approach proposed in $[6,5]$.

Given that $s(k)$ in model (4) is a percentage against the total population, this model can be easily converted into

$$
x_{i}(k+1)=\tilde{b}_{i} \tilde{c}_{i} x_{i}(k)^{2} / x_{i}(0)-\tilde{b}_{i} x_{i}(k)+x_{i}(k) .
$$

Note that the Eq. (5) is only applicable when the following assumptions hold.

1) The lighting project involves a large number of lighting devices such that Eq. (5) is statistically representative for the lighting population decay dynamics.

2) The lighting devices in the $i$ th category are homogeneous and follow the same failure dynamics.

3) The time delay for the individual lighting device installation and commissioning can be ignored.

4) The replacements of the failed lighting devices will not change the lamp population decay dynamics.

\subsection{Control objective and constraints}

For the lighting projects mentioned in Subsection 2.2, PDs will receive different rebate values for installing different types of EE lighting devices, denoted by $R_{i}\left(\mathrm{R}^{1} / \mathrm{kWh}\right)$ on annual basis after the project implementation if the projects are maintained sustainable over the crediting period. However, PDs have to pay for the project transaction cost including the project design, implementation, performance evaluation and maintenance at their own budget. The initial investment $\Theta_{1}$ of the project is estimated by

$$
\Theta_{1}=\sum_{i=1}^{I} \alpha_{i} x_{i}(0)+\beta,
$$

where $\alpha_{i}$ denote the cost related to individual EE lighting device, including the procurement, delivery, removal of an old device and installation of a new device in the $i$ th lighting group; $\beta$ denotes the project transaction cost, usually $\beta$ occupies $10 \%$ of $\Theta_{1}$ and it is a once-off expense per project.

The performance of an energy conservation project is usually quantified by a measurement and verification $(\mathrm{M} \& \mathrm{~V})$ approach $[11,32]$. The lighting project performance is calculated by the product of the number of survived lighting population and the average savings of individual EE lighting unit. As time goes by, the total project rebate will become less and less given that the lighting population decays if the failed EE lighting devices are not replaced. In case no maintenance is carried out, the PDs' benefit is calculated by

$$
\Pi_{1}=\sum_{i=1}^{I} \sum_{k=0}^{K-1} r_{i} \bar{x}_{i}(k)-\Theta_{1},
$$

${ }^{1} \mathrm{R}$ is short for the South African Currency: Rand. The annual average USD to Rand exchange rate in 2013 is $1 \mathrm{USD}=\mathrm{R} 9.65$. where $r_{i}$ is the rebate per EE device in the $i$ th group, $r_{i}=R_{i} E S_{i}$. $E S_{i}$ is the energy saving (in $\mathrm{kWh}$ ) per EE device that is determined by the M\&V approach. For simplicity, it is assumed that both $r_{i}$ and $E S_{i}$ are constant during each sampling interval. $\bar{x}_{i}(k)$ represents the number of survived EE lighting devices in the $i$ th group during the time period $[k, k+1) . \bar{x}_{i}(k+1)$ is calculated by Eq. (5) and

$$
\bar{x}_{i}(k+1)=f_{i}\left(\bar{x}_{i}(k)\right)
$$

As discussed previously, proper replacements of failed lamps contribute to a sustainable project performance, which will consequently increase the PDs' benefit. From PDs' point of view, although the project maintenance brings additional benefits, it requires extra investments. If a number of $u_{i}(k)$ failed EE devices will be replaced during the time interval $[k, k+1)$, then the PDs' benefit is calculated by

$$
\Pi_{2}=\sum_{i=1}^{I} \sum_{k=0}^{K-1}\left[r_{i} x_{i}(k)-\alpha_{i} u_{i}(k)\right]-\Theta_{1},
$$

where $x_{i}(k)$ represents the number of survived EE lamps in the $i$ th group during the time period $[k, k+1)$ and $x_{i}(k)$ is calculated by the state equation in Eq. (1). When replacing the failed EE devices, the additional investment needs to cover the expenses for each replacement, which is calculated by $\alpha_{i} u_{i}(k)$.

With additional investments for a proper project maintenance, the PDs' absolute benefit $\Pi_{2}$ might be greater than $\Pi_{1}$. However, a greater $\Pi_{2}$ does not imply that the project with maintenance is more beneficial than the project without maintenance since this is not a fair-comparison. To ensure a faircomparison, the total project benefit needs to be normalised against the total project investment. This normalised value is called cost-benefit ratio between the total project profit and the total project investment. The cost-benefit ratio $J_{1}$ for the project without maintenance is calculated by $\Pi_{1} / \Theta_{1}$. The cost-benefit ratio $J_{2}$ for the project with maintenance is calculated by $\Pi_{2} / \Theta_{2}$ where

$$
\Theta_{2}=\Theta_{1}+\sum_{i=1}^{I} \sum_{k=0}^{K-1}\left[\alpha_{i} u_{i}(k)\right] .
$$

Therefore, to maximise PDs' benefits, the objective function is to

$$
\min J_{2}=-\frac{\Pi_{2}}{\Theta_{2}} .
$$

The inequality constraints of the OMP problem are given as

$$
\left\{\begin{array}{l}
x_{i}(k) \leq x_{i}(0), \\
x_{i}(k) \geq 0.5 x_{i}(0), \\
\sum_{i=1}^{I} \sum_{j=0}^{k-1}\left[\alpha_{i} u_{i}(j)-r_{i} x_{i}(j)\right] \leq 0,
\end{array}\right.
$$

where the first two constraints indicate that the project population shall be within the boundary of $\left[0.5 x_{i}(0), x_{i}(0)\right]$. The lower bound is designed to guarantee the projects' sustainable performance. The upper bound is a hard constraint since $x_{i}(0)$ is decided by the project scope boundary. The third constraint is the limit of the available budget for the maintenance. In other 
words, the expense for the maintenance at time $k$ must not exceed the cumulative available profits of the project at the end of the time period $[0, k-1)$. Apparently, the requirements of the "full maintenance" may sometimes violate the third constraints.

The OMP problem is then translated into an optimal control problem as follows:

Given the control system dynamics (1), the objective function (9) and the inequality constraints (10), the OMP problem is to find an optimal control sequence $u_{i}(k)$ that minimises $J_{2}$ subject to the equality constraints (1) and inequality constraints (10).

The formulated OMP problem can be directly solved by open loop optimal control techniques when random measurement errors and model uncertainties are negligible. However, due to the unavoidable uncertainties and disturbances coupled in the OMP problem, it is more appropriate to adopt a closed-loop control approach that is robust against the system uncertainties and disturbances to solve the problem.

\section{MPC algorithm to the OMP problem}

This section proposes an closed-loop MPC approach to solve the OMP problem due to its superiority in handling the possible modelling uncertainties and measurement disturbances in the control systems.

The OMP problem in Section 2 is defined over the time interval $[0, K)$ to optimise the control variables $\left[u_{i}(0), u_{i}(1), \ldots, u_{i}(K-1)\right]$. It is obvious that when the same OMP problem is considered over the time interval $[m, m+$ $N), m \in[0, K)$, then the control variables are changed into $\left[\left.u_{i}\right|_{m}(m),\left.u_{i}\right|_{m}(m+1), \ldots,\left.u_{i}\right|_{m}(m+N-1)\right]$. In an MPC approach, a finite-horizon optimal control problem is repeatedly solved and only the first control input is applied to the system. Consider an optimisation horizon with length $N$, the OMP problem over the time interval $[m, m+N)$ can be defined as the following optimisation problem:

$$
\min \tilde{J}_{2}=-\tilde{\Pi}_{2} / \tilde{\Theta}_{2}
$$

subject to state and input constraints

$$
\left\{\begin{array}{l}
\left.x_{i}\right|_{m}(m+h) \leq x_{i}(0), \\
\left.x_{i}\right|_{m}(m+h) \geq 0.5 x_{i}(0), \\
\pi(m)+\sum_{i=1}^{I} \sum_{q=m}^{m+h-1}\left[\left.\alpha_{i} u_{i}\right|_{m}(q)-\left.r_{i} x_{i}\right|_{m}(q)\right] \leq 0, \\
\left.x_{i}\right|_{m}(m+h)=f_{i}\left(\left.x_{i}\right|_{m}(m+h-1)\right)+\left.u_{i}\right|_{m}(m+h-1),
\end{array}\right.
$$

and the terminal constraint

$$
\sum_{i=1}^{I}\left[\left.\alpha_{i} u_{i}\right|_{m}(m+N-1)-\left.r_{i} x_{i}\right|_{m}(m+N-1)\right] \leq 0,
$$

where $h \in[1,2, \ldots, N)$ and the notation $\left.\right|_{m}$ means that the value is obtained based on the information available at time $m$; and

$$
\pi(m)=\sum_{i=1}^{I} \sum_{q=0}^{m-1}\left[\alpha_{i} \bar{u}_{i}(q)-r_{i} x_{i}(q)\right]
$$

denotes the cumulative available profits at the end of the time period $[0, m-1)$, and $\bar{u}_{i}(q)$ 's are the control inputs obtained at time $q$.

$$
\begin{gathered}
\tilde{\Pi}_{2}=\sum_{i=1}^{I} \sum_{h=m}^{m+N-1}\left[\left.r_{i} x_{i}\right|_{m}(h)-\left.\alpha_{i} u_{i}\right|_{m}(h)\right]-\Theta_{1}, \\
\tilde{\Theta}_{2}=\Theta_{1}+\sum_{i=1}^{I} \sum_{h=m}^{m+N-1}\left[\left.\alpha_{i} u_{i}\right|_{m}(h)\right] .
\end{gathered}
$$

Both the objective functions (11) and constraints (12) are nonlinear as the population decay dynamics model in Eq. (5) is nonlinear. The interior-point algorithm is chosen to to find the optimal solutions [24]. The MPC formulation of the OMP problem in (11)-(16) can be solved over the prediction horizon $[m, m+N)$ given the initial condition $x_{i}(m)$. Let the obtained optimal control inputs be denoted by $\left\{\left.\mathbf{u}_{i}^{*}\right|_{m}, i=1,2, \ldots, I\right\}$, then only the optimal solution in the first sampling period $[m, m+1)$ is applied, denoted by $\left.\overline{\mathbf{u}}_{i}\right|_{m}=\left.\mathbf{u}_{i}^{*}\right|_{m}(1)$. According to Eq. (12), the obtained optimal $\left.\overline{\mathbf{u}}_{i}\right|_{m}$ is applied to calculate $\mathbf{x}(m+1)$ and $\mathbf{y}(m+1) \cdot \mathbf{y}(m+1)$ then becomes the initial condition of the MPC formulation over the next prediction horizon $[m+1, m+N+1)$. Thus a closed-loop feedback is obtained. In case of applying the MPC approach on a finite time interval with length $K$, then the optimisation horizon (or control horizon, which is equivalent to prediction horizon in this study) is reduced to $N=K-m$ when $N>K-m$. This process will be repeated until all the optimal control inputs $\overline{\mathbf{u}}$ are obtained over the period $[0, K)$.

For an undisturbed control system model, where the modelling uncertainties $\mathbf{w}(k)$ and measurement disturbances $\mathbf{v}(k)$ are not considered, the system output $\mathbf{y}(k)$ equals the predicted state variable $\mathbf{x}(k)$ and is taken as the initial state for the optimal control problem over the next finite horizon. The above ideas can be formulated as Algorithm 1.

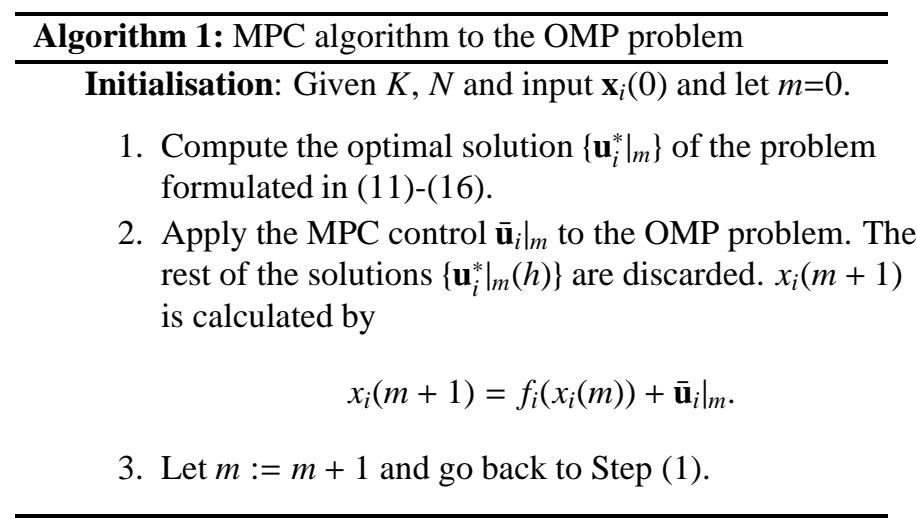

The above MPC algorithm is executed over the entire control period $[0, K)$ to solve the OMP problem.

Assumption 1. Parameters $\alpha_{i}, r_{i}$ and decay function $f_{i}(x)$ satisfy:

$$
0.5 x_{i}(0)-f_{i}\left(0.5 x_{i}(0)\right) \leq \frac{0.5 r_{i} x_{i}(0)}{\alpha_{i}}
$$

Remark 1. Assumption 1 indicates that, if $x_{i}(j)$ happens to reach its lower bound, there always exists a beneficial control $u_{i}=\frac{0.5 r_{i} x_{i}(0)}{\alpha_{i}}$ to guarantee that $x_{i}(j+1)$ is within constraints. 
Proposition 1. Suppose that all parameters satisfy the condition given in Assumption 1. With the MPC Algorithm 1, the closed-loop system possesses the following properties:

1. the optimisation is always feasible, if it is feasible at $k=0$;

2. there are benefits after retrofitting in every step, or namely $\pi(m) \leq 0$.

\section{Proof.}

1. At $k=0$, the optimisation problem (11) is feasible. It will be proved as following that feasibility at $k=j$ implies feasibility at $k=j+1$.

Suppose that, at $k=j$, the optimisation problem (11) is feasible, and its solution can be obtained by

$$
\left.\mathbf{u}_{i}^{*}\right|_{j}=\left[\left.u_{i}^{*}\right|_{j}(j), \cdots,\left.u_{i}^{*}\right|_{j}(j+N-1)\right]^{T} .
$$

The corresponding states are

$$
\left.\mathbf{x}_{i}^{*}\right|_{j}=\left[\left.x_{i}^{*}\right|_{j}(j), \cdots,\left.x_{i}^{*}\right|_{j}(j+N-1)\right]^{T},
$$

where $\left.x_{i}^{*}\right|_{j}(j)=x_{i}(j)$. The above optimal solution and the corresponding states satisfy all constraints given in (12) and (13).

According to Algorithm 1, the first element of $\left.\mathbf{u}_{i}^{*}\right|_{j}$ is implemented; consequently, $x(j+1)=\left.x^{*}\right|_{j}(j+1)$.

Then, at $k=j+1$, select

$$
\begin{gathered}
\left.u_{i}\right|_{j+1}(j+1)=\left.u_{i}^{*}\right|_{j}(j+1), \\
\left.u_{i}\right|_{j+1}(j+2)= \\
=\left.u_{i}^{*}\right|_{j}(j+2), \\
\vdots \\
\left.u_{i}\right|_{j+1}(j+N-1)=\left.u_{i}^{*}\right|_{j}(j+N-1) .
\end{gathered}
$$

It follows that

$$
\begin{gathered}
\left.x_{i}\right|_{j+1}(j+1)=\left.x_{i}^{*}\right|_{j}(j+1), \\
\left.x_{i}\right|_{j+1}(j+2)=\left.x_{i}^{*}\right|_{j}(j+2), \\
\vdots \\
\left.x_{i}\right|_{j+1}(j+N-1)=\left.x_{i}^{*}\right|_{j}(j+N-1) .
\end{gathered}
$$

It is obvious that the above predicted controls and states satisfy constraints (12). Select

$$
\begin{aligned}
& \left.u_{i}\right|_{j+1}(j+N)= \\
& \min \left[\frac{\left.r_{i} x_{i}\right|_{j+1}(j+N)}{\alpha_{i}}, x_{i}(0)-f\left(\left.x_{i}\right|_{j+1}(j+N)\right)\right] .
\end{aligned}
$$

At $k=j+1$ the terminal constraint (13) is satisfied, indicating that the third line of constraints (12) is satisfied.

$$
\begin{aligned}
& \text { If } \frac{\left.r_{i} x_{i}\right|_{j+1}(j+N)}{\alpha_{i}} \leq x_{i}(0)-f\left(\left.x_{i}\right|_{j+1}(j+N)\right) \text {, then } \\
& \left.u_{i}\right|_{j+1}(j+N)=\frac{\left.r_{i} x_{i}\right|_{j+1}(j+N)}{\alpha_{i}} \geq \frac{0.5 r_{i} x_{i}(0)}{\alpha_{i}}
\end{aligned}
$$

assuring that the first two lines of (12) are satisfied. If $\frac{\left.r_{i} x_{i}\right|_{j+1}(j+N)}{\alpha_{i}} \geq x_{i}(0)-f\left(\left.x_{i}\right|_{j+1}(j+N)\right)$, then

$$
\left.u_{i}\right|_{j+1}(j+N)=x_{i}(0)-f\left(\left.x_{i}\right|_{j+1}(j+N)\right),
$$

indicating a full maintenance terminal control that satisfies all constraints.

As a result, at $k=j+1$, it can be found at least one feasible solution

$$
\begin{aligned}
\left.\mathbf{u}_{i}\right|_{j+1} & \triangleq\left[\left.u_{i}\right|_{j+1}(j+1), \cdots,\left.u_{i}\right|_{j+1}(j+N-1),\left.u_{i}\right|_{j+1}(j+N)\right]^{T} \\
& =\left[\left.u_{i}^{*}\right|_{j}(j+1), \cdots,\left.u_{i} *\right|_{j}(j+N-1),\left.u_{i}^{*}\right|_{j}(j+N-1)\right]^{T}
\end{aligned}
$$

that satisfies all constraints in (12) and (13), indicating that the optimisation problem (11) is feasible at $k=j+1$.

According to mathematical induction, the optimisation problem is feasible at all future times, if it is feasible at $k=0$.

2. Given that the optimisation is feasible, the fact that $\pi(m) \leq$ 0 follows directly from the third line of constraint (12).

Remark 2. In this study, the proposed MPC is actually employed to solve an optimisation problem (instead of a control problem). Stability of the closed-loop system is trivial, since system states are always bounded due to constraints (12). Consequently, proofs of feasibility and benefits are sufficient to the optimisation problem.

Remark 3. Robustness is an inherent property of MPC, and it is the very beginning motivation when MPC is firstly invented. $M P C$ is able to detect the effects of disturbances when measuring the current states, and make corresponding compensations. To guarantee better robustness, some variations are introduced to Algorithm 1 as follows.

In practice, the modelling uncertainties and measurement disturbances are unavoidable. For the lighting projects, the predicted system states that refer to the survived lamp population may not be the same as the actual ones. Also, measurement of the survived lamps is done on sampling basis, usually by $\mathrm{M} \& \mathrm{~V}$ inspection bodies, due to the large number of lamps involved. Therefore, the MPC approach developed is applied to a disturbed system with sampled measurement feedback and deals with the uncertainties and disturbances in a closed-loop way. That is, the sampled measurements that are not equal to the actual number of lamps survived are used as feedback information by the controller in optimisation. To demonstrate influences of the uncertainties and disturbances and to verify the MPC method's effectiveness in coping with them, the Algorithm 1 is modified accordingly: In Step (2), the actual state is obtained by

$$
x_{i}(m+1)=f_{i}\left(x_{i}(m)\right)+\left.\overline{\mathbf{u}}_{i}\right|_{m}+w_{i}(m)
$$

and the measurement of the system output

$$
y_{i}(m)=x_{i}(m)+v_{i}(m)
$$

is taken as the true plant state by the MPC controller in the next optimisation horizon to improve the plant performance. The terms $w_{i}(m)$ and $v_{i}(m)$ are simulated by $-\varepsilon_{i}+2 \varepsilon_{i} \delta(m)$, where 
$\delta(m)$ 's are independent and identically distributed random numbers in $[0,1]$ and $\varepsilon_{i}$ 's are the error bounds. Thus an evenly distributed error from $-\varepsilon_{i}$ to $\varepsilon_{i}$ is added on the system states $x_{i}(m)$ during each sampling interval.

\section{Case study}

In this section, an optimal maintenance plan designed for a lighting retrofit project is taken as a case study to illustrate the effectiveness of the proposed optimal control system approach in solving the OMP problem.

A lighting retrofit project is going to be implemented to reduce the lighting load in various residential households in the Northern areas of South Africa. This lighting project is sponsored by a local utility under the national demand side management programme. A large number of energy efficient CFLs and LEDs will be installed to replace existing inefficient incandescent lamps (ICLs) and halogen downlighters (HDLs), respectively. According to the project regulation policies, the removed HDLs and ICLs will be counted, stored and destroyed by a contracted disposal company. The CFLs to be installed have a rated life of 3 years while the LEDs have a rated life of 6 years. The energy efficiency lamps have the equivalent lumen to the replaced old lamps. The adopted CFLs and LEDs are naturally classified into two homogeneous lighting groups as lamps in each group share the same technical specification, same working condition and operating schedule. Therefore, the same lamp population decay dynamics can also be observed and modelled in each lighting group.

PDs are encouraged to implement the project at their own cost and different rebate rates will be offered by the project sponsor to different lighting technologies. Since the unit retrofit price of an LED is more expensive than that of a CFL, the PDs will receive a higher rebate rate from the installations of LEDs. The project qualifies a crediting period of 10 years, during which PDs can receive their rebates on annual basis if the population of the newly installed EE lighting devices are properly maintained. If more than $50 \%$ of one kind of lamps is malfunctioned, then the project rebate will be ceased. The project performance in terms of energy savings will be reported at the end of each crediting year by a third-party $M \& V$ inspection company. The number of survived lamps will also be inspected by sampling and surveys at each reporting interval. Once lamp failures are observed, PDs' are allowed to replace some (or all) of the failed EE devices at the end of each crediting year to avoid the cease of project rebates. More project details that obtained from the project participants are listed in Table 1.

In order to obtain an optimal maintenance plan for the abovementioned lighting project, the optimal control sequences $u_{i}(k)$ need to be identified by the MPC algorithms that are introduced in Section 3 with the application of the initial conditions of the parameters appear in (11)-(16). The relevant initial values are listed in Table 1. As discussed, the periodic PM maintenance policy is applied to this lighting project. For this study, the maintenance intervals are decided to be one year in order to align with the annual project performance reporting by the $\mathrm{M} \& \mathrm{~V}$ practitioners. The advantage is that the latest sampled
Table 1: Information of the lighting project

\begin{tabular}{lll}
\hline Parameters & CFL group & LED group \\
\hline Initial population & $x_{1}(0)=404876$ & $x_{2}(0)=207693$ \\
Unit retrofit price & $\alpha_{1}=\mathrm{R} 32$ & $\alpha_{2}=\mathrm{R} 260$ \\
Daily burning hours & $O_{1}=5 \mathrm{~h}$ & $O_{2}=10 \mathrm{~h}$ \\
Power of old lamps & $P_{1}=60 \mathrm{~W}$ & $P_{2}=35 \mathrm{~W}$ \\
Power of EE lamps & $\hat{P}_{1}=14 \mathrm{~W}$ & $\hat{P}_{2}=4 \mathrm{~W}$ \\
Rebate per kWh & $R_{1}=\mathrm{R} 0.42$ & $R_{2}=\mathrm{R} 0.55$ \\
Coefficient 1 & $\tilde{b}_{1} \tilde{c}_{1}=0.7478$ & $\tilde{b}_{2} \tilde{c}_{2}=0.8936$ \\
Coefficient 2 & $\tilde{c}_{1}=0.8553$ & $\tilde{c}_{2}=0.9201$ \\
\hline
\end{tabular}

and surveyed lamp survival/failure rate of the lighting project population is available as the feedback signals of the control system.

The coefficients in the population decay dynamics model (5) are identified by the system identification approach proposed in $[6,5]$ and also provided in Table 1 . The annually sampled lamp population decay patterns are presented in Fig. 1, where the horizontal axis indicates the project crediting years and the vertical axis shows the survived lamp population. Obviously, without proper maintenance, the lamp population decreases very fast to zero as time goes by, which will cause a cease of project rebate.

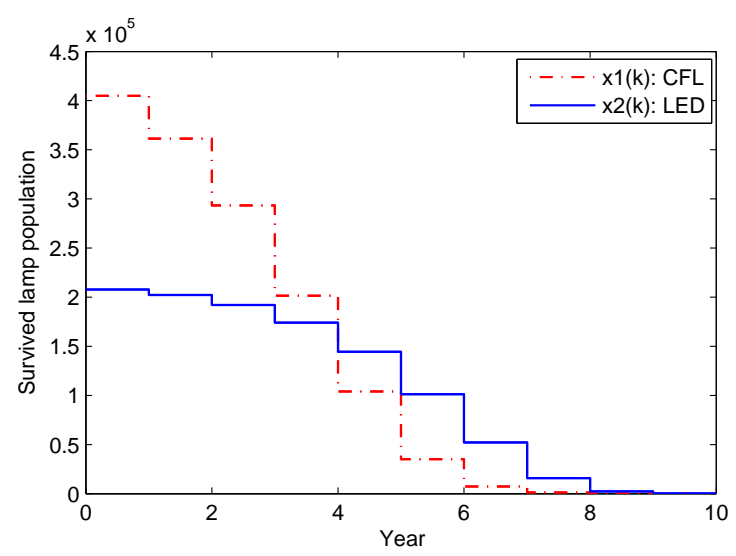

Figure 1: Survived lamp population without maintenance.

For this case study, all computations are carried out by the Matlab program. In particular, the optimal control inputs are computed by the "fmincon" code of the Matlab Optimisation Toolbox [33]. The optimisation settings of the "fmincon" function are shown in Table 2, where the interior-point algorithm is chosen as the optimisation algorithm; the three termination tolerances on the function value, the constraint violation, and the design variables are also given. In addition, "fmincon" calculates the Hessian by a limited-memory, large-scale quasiNewton approximation, where 20 past iterations are remembered. Besides these settings, a search starting point and the boundaries of the control variable are also assigned. For the MPC approach, the optimisation horizon $N$ is chosen as 5 years.

The computation results are presented in Figs. 2-3 and Table 3. In the Figs. 2-3, the horizontal axes indicate the project crediting years and the vertical axes show the survived lamp 


\begin{tabular}{ll}
\multicolumn{2}{l}{ Table 2: Optimisation settings. } \\
\hline Categories & Options \\
\hline Algorithm & interior-point \\
TolFun & $10^{-45}$ \\
TolCon & $10^{-45}$ \\
TolX & $10^{-45}$ \\
Hessian & 'lbfgs', 20 \\
$l b:$ & 0 \\
$u b:$ & $x_{1}(0)+x_{2}(0)$ \\
$u_{i}(0):$ & 1000 \\
\hline
\end{tabular}

population. The solid lines (in blue) denote the system states of the annual survived lamps over the crediting period. The dash-dotted line (in black) denotes the survived lamp population without control/maintenance. The stem lines with a circle (in red) denote the number of failed lamps to be replaced over the 10-year crediting period. As shown by the solid lines (in blue), lamp failures are identified at the end of each year, then a number of these failed devices will be replaced as denoted by the stem lines. The optimal control strategy in the CFL group tends to maintain the lamp population to the full population over the entire crediting period. However, no failed LEDs are going to be replaced between the 7 th and 10th year.

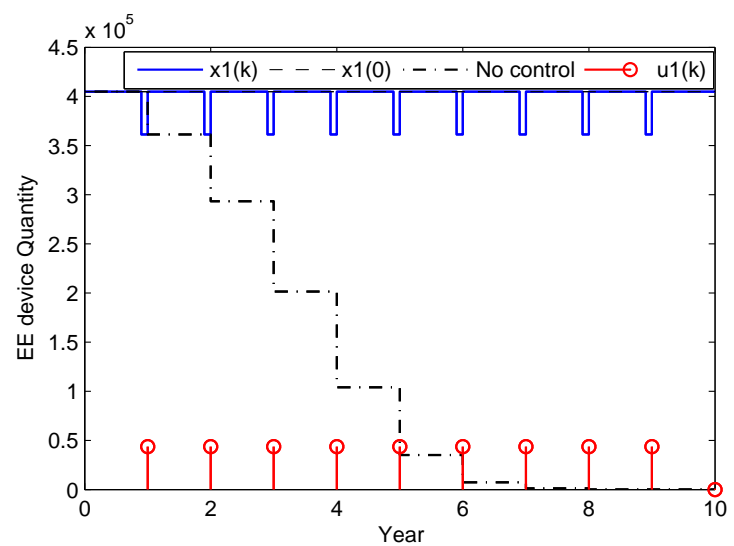

Figure 2: Optimal control strategy for the CFL group.

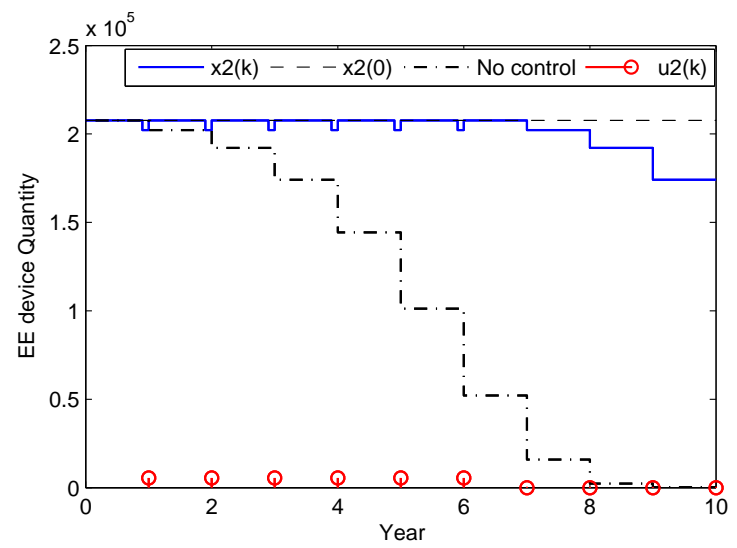

Figure 3: Optimal control strategy for the LED group.
The key performance indicators (KPI), such as the total investments (in million Rand (MR)), total profits (in MR), the cost-benefit ratio, and the total energy savings (in $\mathrm{MWh}$ ) for the lighting retrofit project under the scenarios with no maintenance (NM), full maintenance (FM), and optimal maintenance strategies are calculated and summarised in Table 3. These key performance indicators in Table 3 are calculated without considering the control system disturbances and uncertainties. The comparison of the performance between no maintenance and optimal maintenance strategies indicates that the energy savings increase by $140 \%$ with the optimal maintenance strategy. In addition, PDs receive $279 \%$ more profits with an extra $28 \%$ investment for the project maintenance. As commented in [7], a cost-benefit ratio above one indicates a beneficial programme and a higher cost-benefit ratio implies better financial benefits to the PDs. Thus the lighting project without maintenance is not beneficial to the PDs as the cost-benefit ratio 0.7148 is below one. When comparing the performance between the full maintenance and optimal maintenance strategies, it is observed that with the optimal maintenance strategy, the total project investment is $7 \%$ less while the total project profit is $2 \%$ more than the same performance indicators under the full maintenance strategy. It is also found the cost-benefit ratio of the optimal maintenance strategy is $9 \%$ greater than that of the full maintenance strategy. Although the project energy savings with the full maintenance strategy is $1 \%$ higher than that of the optimal maintenance strategy, there are potential risks that the full maintenance strategy cannot be physically implemented due to the PDs' budget constraints.

As discussed in [33], the MPC algorithm is robust against the control system uncertainties and disturbances, which exhibits better performance than the open loop optimisation approach. In practice, the modelled or measured control system states may not be exactly the same as the actual system states due to the unavoidable modelling and measurement uncertainties. In order to test the performance of the MPC algorithm in dealing with the uncertainties and disturbances, an evenly distributed error is added on the measured system states and a system output feedback is also employed in the MPC approach. The error bands of the random noises are $\pm 1 \% x_{i}(k)$ in each lighting group given the large scale of the lighting project population.

Table 4: MPC v.s. open loop optimal solutions.

\begin{tabular}{lcc}
\hline Key performance indicators & MPC & Open loop \\
\hline Total investment & 95.868 & 95.504 \\
Total profit & 201.280 & 198.030 \\
Cost-benefit Ratio & 2.0995 & 2.0735 \\
Energy saving & 636580 & 629970 \\
\hline
\end{tabular}

The project key performance indicators calculated with uncertainties by both the MPC approach and the open loop approach are given in Table 4. If the uncertainties were not revealed and handled by the MPC approach, then the project key performance indicators would have been calculated by applying the open loop optimal solutions directly to the scenario. Comparing the performance indicators in Table 4 , the results 
Table 3: Project key performance indicator analysis.

\begin{tabular}{lccccc}
\hline Key performance indicators & NM & FM & OM & OM v.s. NM & OM v.s. FM \\
\hline Total investment & 74.396 & 102.61 & 95.507 & $28 \%$ & $-7 \%$ \\
Total profit & 53.180 & 197.95 & 201.650 & $279 \%$ & $2 \%$ \\
Cost-benefit Ratio & 0.7148 & 1.9293 & 2.1113 & $195 \%$ & $9 \%$ \\
Energy saving & 265500 & 642880 & 636690 & $140 \%$ & $-1 \%$ \\
\hline
\end{tabular}

NM: no maintenance; FM: full maintenance; OM: optimal maintenance; v.s.: versus.

from MPC approach exhibits better economic benefits and energy savings. This verifies the advantageous performance of the MPC approach for the OMP problem against other open loop optimisation approaches. It also reveals that the MPC approach with the system output feedback is able to handle the control system uncertainties.

\section{Simulations on model applications}

The case study in Section 4 successfully demonstrates the advantageous performance of the proposed approach. In order to test applicability of the proposed model for the OMP design of similar lighting projects, the model performances in terms of the project cost-benefit ratios are calculated and compared under three scenarios:

1) Model performance v.s. rebate tariff;

2) Model performance v.s. lighting life span;

3) Model performance v.s. lighting unit retrofit price.

The simulation results are presented in three subsections as follows.

\subsection{Model performance versus rebate tariff}

For lighting retrofit projects registered under different energy conservation programmes, the rebate tariffs may be different. In the case study, the rebate tariff is $R_{e}=\{0.42,0.55\}$, which represents $\mathrm{R} 0.42 / \mathrm{kWh}$ savings for CFL retrofits and $\mathrm{R} 0.55 / \mathrm{kWh}$ savings for LED retrofits. In order to investigate the model performance against different rebate tariffs, a simulation is carried out as follows. The maximum project cost-benefit ratio is calculated by the introduced MPC approach with $R_{e}=\{0.42,0.55\}$ as a reference. In the simulation, $R_{e}$ is changed by $\pm 10 \%, \pm 20 \%$, and $\pm 50 \%$. The model performance indicators are calculated each time when $R_{e}$ changes. The simulation results are shown and compared in Fig. 4. It is observed that the project performance drops when the rebate tariff decreases. Moreover, for a given rebate tariff, the project cost-benefit ratios calculated by the optimal maintenance, full maintenance, and no maintenance strategies are in the first, second, and third places, respectively. This observation is consistent with the conclusions draw from the case study. It is also noted that when the rebate tariff drops by $50 \%$, the project cost-benefit ratio becomes negative. The control inputs of the optimal maintenance strategies are exactly the same as presented in Figs 2-3.

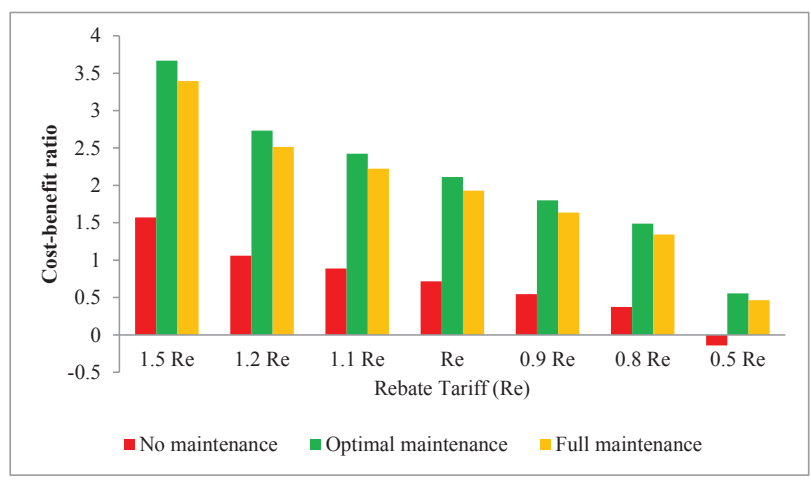

Figure 4: Model performance versus rebate tariff.

\subsection{Model performance versus unit retrofit price}

The unit retrofit prices may be different for different lighting retrofit projects. In the case study, the unit retrofit price is denoted by $P_{r}=\{32,260\}$, which represents R 32 per CFL retrofit and R 260 per LED retrofit. In order to investigate the model performance against different unit retrofit prices, a simulation is carried out as follows. The maximum project cost-benefit ratio with $P_{r}=\{32,260\}$ is calculated by the introduced MPC approach as a reference. In the simulation, $P_{r}$ is changed by $\pm 10 \%, \pm 20 \%$, and $\pm 50 \%$. The model performance indicators are calculated each time when $P_{r}$ changes. The simulation results are shown and compared in Fig. 5. It is observed that the project performance drops when the unit retrofit price increases. Moreover, for a given rebate tariff, the project costbenefit ratios calculated by the optimal maintenance, full maintenance, and no maintenance strategies are in the first, second, and third places, respectively. This observation is consistent with the conclusions draw from the case study. The control inputs of the optimal maintenance strategies are exactly the same as presented in Figs 2-3.

\subsection{Model performance versus lighting life span}

As discussed in Subsection 2.3, life span determines the lighting population decay dynamics. Lighting devices with various life spans may be involved in different lighting retrofit projects. In the case study, the average lighting device life span is denoted by $L_{i}=\{3,6\}$, which represents an average life span of 3 years for CFLs and 6 years for LEDs. In order to investigate the model performance against different lighting life spans, a simulation is carried out as follows. The maximum project costbenefit ratio is calculated by the introduced MPC approach with 


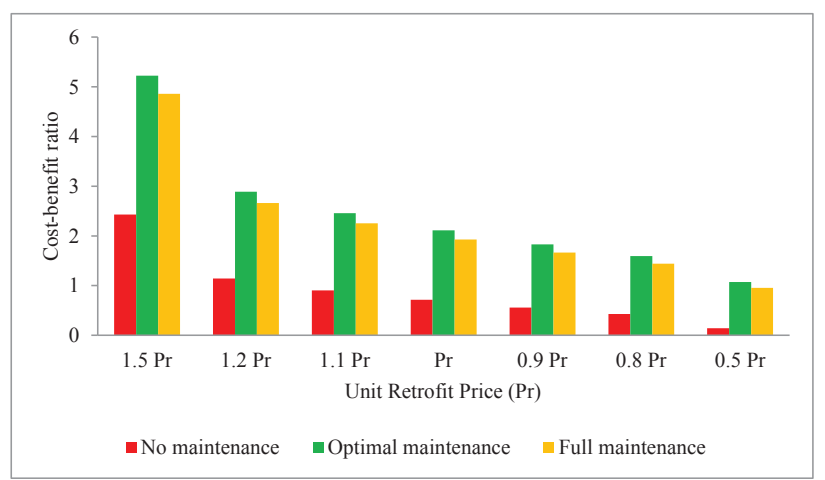

Figure 5: Model performance versus unit retrofit price.

$L_{i}=\{0.42,0.55\}$ as a reference. In the simulation, $L_{i}$ is changed by $\pm 10 \%, \pm 20 \%$, and $\pm 50 \%$. The model performance is calculated each time when $L_{i}$ changes. The simulation results are shown and compared in Fig. 6. It is observed that the project performance drops when the device life span decreases. Moreover, for a given rebate tariff, the project cost-benefit ratios calculated by the optimal maintenance, full maintenance, and no maintenance strategies are in the first, second, and third places, respectively. This observation is consistent with the conclusions draw from the case study.

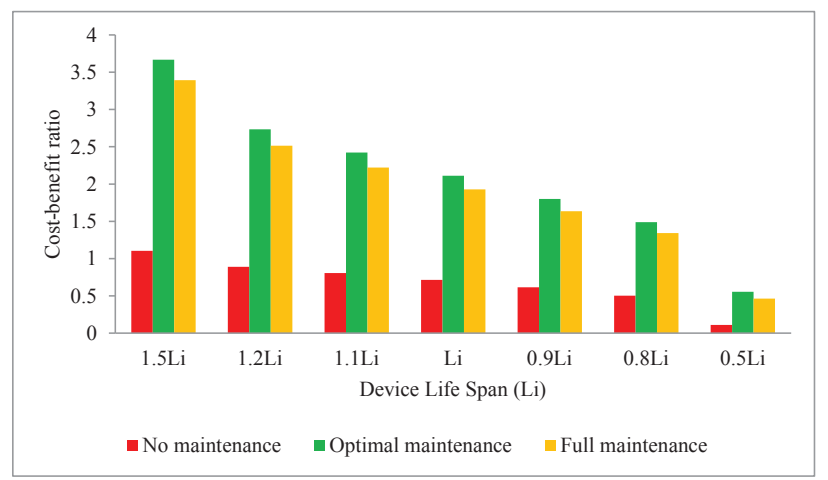

Figure 6: Model performance versus device life span.

The control inputs of the optimal maintenance strategies for lighting projects with various life spans are presented in Figs 78. In both figures, it is observed that less lamps need to be replaced for lighting projects with longer lighting life spans. For the CFL group, the optimal solutions tend to apply the "full maintenance" strategy. But for the LED group, specific optimal strategies are recommend for lighting projects with different lighting life spans.

\section{Remarks and future work}

The major contributions of this study can be summarised as follows: 1) to formulate the optimal maintenance planning problem into the control system framework, whereby the classic control theories such as optimal control and MPC can be easily applied to solve the maintenance planning problems for the energy efficiency lighting projects. 2) the maximised lighting project performance and PDs' profits in the case study clearly illustrates the advantageous performance of the optimal maintenance strategies to the lighting projects. 3) The proposed control system approach in solving the OMP problem will be widely applicable to other similar projects. The results presented in this study will surely contribute to improvements of the energy efficiency project plans and programme regulations.

This work is also worth of further improvements from the following aspects: 1) The optimal maintenance strategy is determined under the periodic group PM maintenance policies. Obviously, the optimal maintenance plans can also be designed under other applicable maintenance policies, such as the agedependent PM policy, periodic PM policy, failure limit policy, sequential PM policy, repair limit policy, opportunistic maintenance policy, or any policies established as combinations of the aforementioned maintenance policies. 2) The optimal maintenance strategy can be expanded to be more general and applicable for EE projects with more than two lighting groups or with other technologies such as air-conditioning systems or water heating systems once the population decay dynamics are identified. 3) It is also interesting to explore the optimal maintenance policy design over longer project crediting periods or infinite project crediting periods.

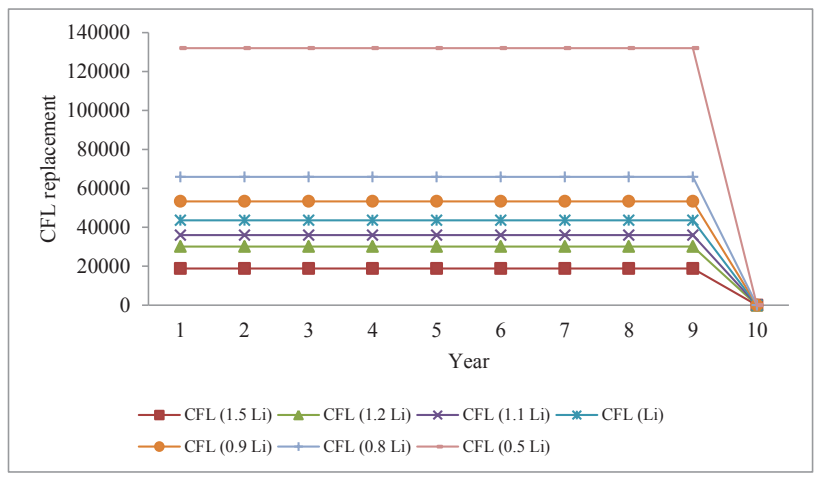

Figure 7: CFL replacements versus device life span.

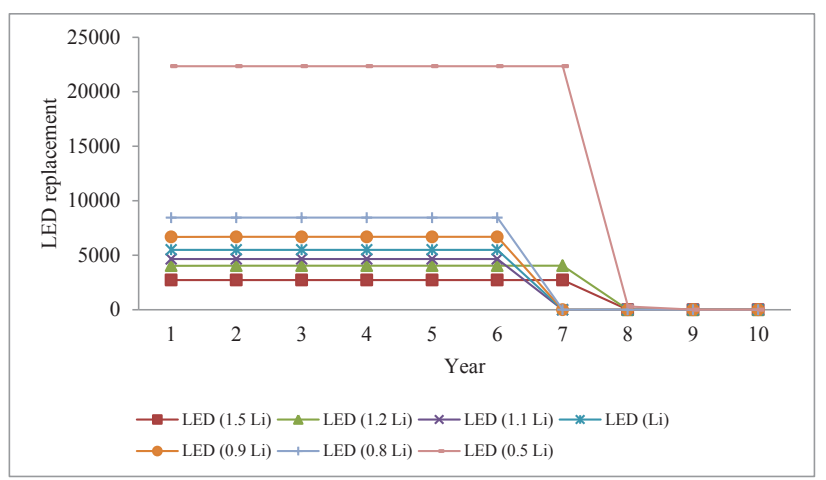

Figure 8: LED replacements versus device life span. 


\section{Acknowledgement}

The authors would like to express our sincere appreciation to the anonymous reviewers for their valuable comments and suggestions. This work is supported by the Centre of New Energy Systems and the National Hub for the Postgraduate Programme in Energy Efficiency and Demand Side Management at the University of Pretoria.

\section{Reference}

[1] Bertoldi, P., \& Rezessy, S. (2008). Tradable White Certificate schemes: fundamental concepts. Energy Efficiency, 1, 237-255.

[2] Botha-Moorlach, M., \& Mckuur, G. (2009). A Report on the factors that influence the demand and energy savings for compact fluorescent lamp door-to-door rollouts in South Africa. Technical Report.

[3] Boukas, E.-K., \& Haurie, A. (1990). Manufacturing flow control and preventing maintenance: a stochastic control approach. IEEE Transactions on Automatic Control, 35, 1024-1031.

[4] Boukas, E. K., \& Yang, H. (1996). Optimal control of manufacturing flow and preventive maintenance. IEEE Transactions on Automatic Control, $41,881-885$.

[5] Carstens, H., Xia, X., \& Ye, X. (2014). Improvements to longitudinal clean development mechanism sampling designs for lighting retrofit projects. Applied Energy, 126, 256 - 265.

[6] Carstens, H., Xia, X., Zhang, J., \& Ye, X. (2013). Characterising compact fluorescent lamp population decay. Mauritius: IEEE AFRICON 2013.

[7] Davis, G. (2002). California standard practice manual: economic analysis of demand-side programs and projects. Technical Report.

[8] Department of Defense Washington DC (1981). Military standard: definitions of term for reliability and maintainability. Technical Report MILSTD-721C Department of Defense Washington DC.

[9] Eskom (2011). The measurement and verification guideline for demandside management projects. Technical Report.

[10] Etschmaier, M. M. (1980). Fuzzy controls for maintenance scheduling in transportation systems. Automatica, 16, $255-264$.

[11] Efficiency Valuation Organization (EVO) (2012). International performance measurement and verification protocol: concepts and options for determining energy and water savings, Volume 1. Technical Report.

[12] International Energy Agency (2006). Light's Labours' Lost-Policies for Energy-efficient Lighting. Paris: OECD/IEA.

[13] Junca, M., \& Sanchez-Silva, M. (2013). Optimal maintenance policy for a compound poisson shock model. IEEE Transactions on Reliability, 62, $66-72$.

[14] Kess, P. (1993). A holistic control philosophy for the process industries. Control Engineering Practice, 1, 835 - 844.

[15] Lewin, D. (1995). Predictive maintenance using PCA. Control Engineering Practice, 3, 415 - 421.

[16] Mahlia, T., Said, M., Masjuki, H., \& Tamjis, M. (2005). Cost-benefit analysis and emission reduction of lighting retrofits in residential sector. Energy and Buildings, 37, 573 - 578.

[17] Michaelowa, A., \& Jotzo, F. (2005). Transaction costs, institutional rigidities and the size of the clean development mechanism. Energy Policy, 33, 511-523.

[18] Mills, E. (2002). Global lighting energy savings potential. Light $\mathcal{F}$ Engineering, 10, 5-10.

[19] Mozzo, M. A. (1999). Measurement and verification of savings in performance contracting. Energy Engineering, 96, 33-45.

[20] Mundaca, L. (2007). Transaction costs of Tradable White Certificate schemes: The energy efficiency commitment as case study. Energy Policy, 35, 4340-4354.

[21] Navigant Consulting (1999). Evaluation of the IFC/GEF Poland efficient lighting project CFL subsidy program. Final report, Edition 2 Netherlands Energy Efficient Lighting B.V. International Finance Corporation/Global Environment Facility.

[22] Pham, H., \& Wang, H. (1996). Imperfect maintenance. European Journal of Operational Research, 94, 425-438.

[23] Qin, S., \& Badgwell, T. A. (2003). A survey of industrial model predictive control technology. Control Engineering Practice, 11, 733 - 764.
[24] Rao, S. S. (2009). Engineering Optimization: Theory and Practice. (4th ed.). Hoboken, New Jersey: John Wiley \& Sons, Inc.

[25] UNFCCC (2007). Approved baseline and monitoring methodology AM0046, Distribution of efficient light bulbs to household. Version 02, Technical Report.

[26] UNFCCC (2010). Approved small scale methodology AMS II.J, Demandside activities for efficient lighting technologies. Version 04, Technical Report.

[27] UNFCCC (2011). Approved small scale methodology AMS II.L, Demandside activities for efficient outdoor and street lighting technologies. Version 01, Technical Report.

[28] UNFCCC (2012). Approved small scale methodology AMS II.N, Demandside energy efficiency activities for installation of energy efficient lighting and or controls in buildings. Version 01.0, Technical Report.

[29] UNFCCC (2012). Project design document form: Gauteng, Free States, Mpumalanga, Limpopo, $\mathcal{G}$ Northern Cape CFL Replacement Project (1) in South Africa. Version 06, Technical Report. Available at: http://cdm.unfccc.int/Projects/DB/DNV-CUK1348741091.78/view.

[30] UNFCCC (2012). Project design document form: The Lebanese CFL Replacement CDM Project - Mount Lebanon. Version 04, Technical Report. Available at: http://cdm.unfccc.int/Projects/DB/DNVCUK1348219106.67/view.

[31] Wang, H. (2002). A survey of maintenance policies of deteriorating systems. European Journal of Operational Research, 139, 469-489.

[32] Xia, X., \& Zhang, J. (2013). Mathematical description for the measurement and verification of energy efficiency improvement. Applied Energy, $111,247-256$.

[33] Xia, X., Zhang, J., \& Elaiw, A. (2011). An application of model predictive control to the dynamic economic dispatch of power generation. Control Engineering Practice, 19, 638-648. 